\title{
MOPSO-based multi-objective TSO planning considering uncertainties
}

\author{
Wang, Qi; Zhang, Chunyu; Ding, Yi; Østergaard, Jacob
}

Published in:

Proceedings of IEEE PES ISGT Europe 2014:

Link to article, DOI:

10.1109/ISGTEurope.2014.7028762

Publication date:

2014

Document Version

Early version, also known as pre-print

Link back to DTU Orbit

Citation (APA):

Wang, Q., Zhang, C., Ding, Y., \& Østergaard, J. (2014). MOPSO-based multi-objective TSO planning considering uncertainties. In Proceedings of IEEE PES ISGT Europe 2014: IEEE.

https://doi.org/10.1109/ISGTEurope.2014.7028762

\section{General rights}

Copyright and moral rights for the publications made accessible in the public portal are retained by the authors and/or other copyright owners and it is a condition of accessing publications that users recognise and abide by the legal requirements associated with these rights.

- Users may download and print one copy of any publication from the public portal for the purpose of private study or research.

- You may not further distribute the material or use it for any profit-making activity or commercial gain

- You may freely distribute the URL identifying the publication in the public portal 


\title{
MOPSO-based Multi-objective TSO Planning Considering Uncertainties
}

\author{
Qi Wang, Member, IEEE, Chunyu Zhang, Member, IEEE, Yi Ding, Member, IEEE, Jacob Østergaard, Senior Member, \\ IEEE \\ Center for Electric Power and Energy \\ Technical University of Denmark \\ Copenhagen, Denmark
}

\begin{abstract}
The concerns of sustainability and climate change have posed a significant growth of renewable energy associated with smart grid technologies. Various uncertainties are the major problems need to be handled by transmission system operator (TSO) planning. This paper mainly focuses on three uncertain factors, i.e. load growth, generation capacity and line faults, and aims to enhance the transmission system via the multi-objective TSO planning (MOTP) approach. The proposed MOTP approach optimizes three objectives simultaneously, namely the probabilistic available transfer capability (PATC), investment cost and power outage cost. A two-phase MOPSO algorithm is employed to solve this optimization problem, which can accelerate the convergence and guarantee the diversity of Pareto-optimal front set as well. The feasibility and effectiveness of the proposed multi-objective planning approach has been verified by the 77-bus system.
\end{abstract}

Index Terms - multi-objective TSO planning; uncertainties; smart grid, two-phase MOPSO

\section{INTRODUCTION}

In Denmark and other European Union (EU) countries, the rapid growth of renewable energies, such as wind and photovoltaic, will prompt critical uncertainty issues on transmission grid. Especially in the future smart grid environment, the supply-side intermittent recourses and demand side stochastic consumptions will test the bearing capacity of the transmission network. The TSO has to explore new ways to circumvent these complex uncertainties.

Due to these emerging new challenges in the power system, ignoring uncertainties in the transmission expansion planning process may result in a huge cost for load shedding or generation dispatching. Recently, the multi-objective planning approach has become more necessary in order to take into account new problems caused by the competitive environment [1]. A number of literatures have been accomplished in multi-objective TSO planning (MOTP) approach. A detailed review of various methods proposed for MOTP can be found in [2-5]. Probabilistic tools, e.g. Monte Carlo simulation [2], are very prevalent for modeling uncertainties in decision making process. Normally, the random uncertainties are noted as probabilistic density function (PDF) while the non-random uncertainties are modeled with scenario technique [6-7]. Besides, fuzzy decision making [7] and fuzzy decision analysis have been used to handle the substantial uncertainties in the future generation and load patterns in a complex environment. These approaches usually need repetitive and huge complicated computational work, which leads to time consuming and boring tasks. Therefore, only a few papers [1-4] take uncertainties into account in MOTP model, which so far has not been considered seriously.

However, the solution of the MOTP problem is almost impossible to be settled by pure mathematical optimization approach, in view of it is a non-convex and nonlinear mixed integer problem. Currently, several intelligent algorithms have been applied to enhance the performance of the decision making problem, including genetic algorithms (GA), particle swarm optimization (PSO), and evolutionary optimization. PSO is one of the most widely used multi-point search algorithms with stochastic behavior and powerful searching ability. PSO was originally developed by Kennedy and Eberhart, who derived the biological inspiration from behavioral models of swarming animals such as bird flocks and fish schools [8]. Based on single-objective PSO a variety of algorithms have been developed to adapt PSO to optimization problems with multiple objectives, referred to as Multi-Objective Particle Swarm Optimization (MOPSO) algorithms. Some of the most common MOPSO variations are presented in [9-11]. Note that, the most famous Sigma method [10] is not capable of achieving good convergence and uniform diversity simultaneously. Therefore, the performance of the MOPSO algorithm needs to be improved to meet the MOTP interest.

In this paper, the probability models of the uncertainties caused by load growth, generation capacity and line faults are formulated. Furthermore, a MOTP approach is proposed to optimize three objectives: the probabilistic available transfer capability (PATC), the cost of power outage and the cost of network investment. Accordingly, a two-phase MOPSO is introduced to accelerate the convergence and guarantee the

This task supported by the Danish national project iPower 
diversity of Pareto-optimal front set. Case study based on the 77-bus system is conducted to demonstrate the feasibility and effectiveness of both the proposed MOTP approach and the improved MOPSO algorithm.

\section{PROBLEM FORMULATION}

\section{A. Uncertaities modeling}

This paper mainly focuses on three major uncertainties, which come from the load growth, generation capacity and line faults, are described and simulated with random variables.

\section{1) Load growth uncertainty modeling}

In terms of the vast spread of new types of demand, for instance, Heat Pump and Electric Vehicle, it will be much more crucial for the TSO to preview the possible consequences of the load volatility.

A probability model based on the normal distribution is introduced to represent the uncertainty of load growth. For existing a load node $i$, assuming that the point of the original load is $P_{i 0}$, the load deviation is $\Delta P_{i}$ can be depict as a random variable, which obeys the normal distribution, as shown in (1). Then, the load of node $i$ is $P_{i}=P_{i 0}+\Delta P_{i}$.

$$
\Delta P_{i} \square N\left(\mu_{i}, \sigma_{i}^{2}\right)
$$

Where

$\mu_{i} \quad$ the expectation of the load based on the forecasting result

$\sigma_{i} \quad$ the corresponding standard deviation.

\section{2) Generation capacity uncertainty modeling}

In the planning stage, a generation node imposed by wind or solar may become a new fluctuation node. TSO has to consider both the generators to be installed and the retirement time of existing generators, and the decision should cover the unpredictable factors such as weather, fossil price, and the change of market rules. The generation capacity node $i$ could follow the discrete probability distribution,

$$
\operatorname{Pr}\left(X=P_{G i k}\right)=a_{i k}
$$

where

$P_{G i k} \quad$ the possible value of generation capacity

$a_{i k} \quad$ the probability of the generation capacity may occur,

$$
0<a_{i k}<1, \quad \sum_{k=1}^{N} a_{i k}=1, \quad k=1,2, \cdots, N .
$$

\section{3) Line faults uncertainty modeling}

Identification of candidate lines usually starts from deciding the planning horizon and performing market forecasting correspondingly. Market simulations can be conducted to examine system stability and reliability, and to identify locations that potentially need new branches.

Furthermore, eliminate a portion of candidate lines by examining their investment cost and construction time. Some lines may also be abandoned if they violate environmental criterion or government policies [1]. Then, using the (0-1) distribution model to represent the uncertainty of the transmission line, where 0 indicates that the line is in failure (or maintenance) status, 1 indicates the line is the normal operating state.

Therefore, each power system scenario of load, generation and transmission line can be generated by Monte Carlo simulation stochastically.

\section{B. MOTP model}

Three indices, namely the PATC, the cost of power outage and the network investment cost, are considered as optimization objectives to implement and achieve three planning goals simultaneously. They, respectively, represent efficiency, reliability and economic for the transmission expansion planning. The proposed model is shown in objectives (3)-(5) and constraints (6)-(19).

\section{Objectives}

$$
\begin{aligned}
& \text { Min } O_{P A T C}=-E\left(\mathbf{e}^{T} \mathbf{d}\right) \\
& \operatorname{Min} O_{O C}=T \sum_{i \in \Omega} I_{E R i} \mathrm{EENS}_{i} \\
& \operatorname{Min} O_{I C}=\sum_{(i, j) \in \Omega} c_{i j} n_{i j}+p \times \Delta W
\end{aligned}
$$

s.t.

$$
\begin{aligned}
& \mathbf{S}^{T} \mathbf{f}_{\mathbf{1}}+\mathbf{g}_{\mathbf{1}}+\mathbf{r}_{\mathbf{1}}=\mathbf{l} \\
& f_{i j 1}-\gamma_{i j}\left(n_{i j}^{0}+n_{i j}\right)\left(\theta_{i 1}-\theta_{j 1}\right)=0 \\
& \operatorname{Pr}\left(\left|f_{i j 1}\right| \leq\left(n_{i j}^{0}+n_{i j}\right) k_{i j} \bar{f}_{i j}\right) \geq \alpha \\
& \mathbf{0} \leq \mathbf{g}_{\mathbf{1}} \leq \overline{\mathbf{g}} \\
& \mathbf{0} \leq \mathbf{r}_{\mathbf{1}} \leq \mathbf{l} \\
& \mathbf{S}^{T} \mathbf{f}_{\mathbf{2}}+\mathbf{g}_{\mathbf{2}}+\mathbf{r}_{\mathbf{2}}=\mathbf{l}+\mathbf{d} \\
& f_{i j 2}-\gamma_{i j}\left(n_{i j}^{0}+n_{i j}\right)\left(\theta_{i 2}-\theta_{j 2}\right)=0 \\
& \left|f_{i j 2}\right| \leq\left(n_{i j}^{0}+n_{i j}\right) \bar{f}_{i j} \\
& \mathbf{0} \leq \mathbf{g}_{\mathbf{2}} \leq \overline{\mathbf{g}} \\
& \mathbf{0} \leq \mathbf{r}_{\mathbf{2}} \leq \mathbf{l}+\mathbf{d} \\
& E\left(\mathbf{e}^{T} \mathbf{d}\right) \geq h \\
& \operatorname{Pr}\left(\mathbf{e}^{T} \mathbf{d} \geq h\right) \geq \beta \\
& 0 \leq n_{i j} \leq \bar{n}_{i j} \\
& \mathbf{d} \geq \mathbf{0}
\end{aligned}
$$

where subscript ${ }_{1}$ means in normal operation conditions, while subscript $_{2}$ represent considering PATC.

$E \quad$ expected value of the operator

e unit vector

$T \quad$ load equivalent duration

$I_{\mathrm{ER} i} \quad$ cost rate of power outage for node $i$ 
EENS $_{i}$ the expected energy not supplied

$c_{i j} \quad$ cost of single line added to the branch $i-j$

$p \quad$ penalty factor

$\Delta W \quad$ total load shedding in operation condition

S the node-branch incidence matrix

f the vector of active power flows

$\mathbf{r}$ the vector of node load shedding

l the vector of forecasted loads

d the vector of active powers growth considering PATC

$\mathbf{g}, \overline{\mathbf{g}}$ the vector of generated active powers, and the upper bound

$f_{i j}, \overline{f_{i j}} \quad$ the active power flow in single line of branch $i-j$, and the upper bound

$k_{i j} \quad$ the load rate of active power flow in single line

$\gamma_{i j}$ the susceptance of single line in the branch $i-j$

$n_{i j}^{0}, n_{i j}, \overline{n_{i j}}$ the number of existing lines, added lines of branch

$i-j$, and the upper bound

$\theta_{i}, \theta_{j} \quad$ the voltage angle of node $i, j$

$h$ the expected value of the operator considering PATC

$\alpha \quad$ the pre-set confidence in normal operation conditions

$\beta \quad$ the pre-set confidence considering PATC

$\Omega \quad$ the set of the buses of the existing and new added lines

EENS $_{i}$ is the expected energy not supplied. The total number of load loss events is calculated according to the " $N$ 1 " and " $N-2$ " line outage scenarios [12].

\section{Methodology}

\section{A. Single Objective PSO}

The single objective PSO optimizes one objective function via the population-based stochastic tracking, where particle denotes the potential optimal solution and swarm covers a set of particles. Each particle moves through a multiple dimensions space to pursue a possible solution by conducting self and neighbors' experiences. In the searching space, the behavior of a particle can be recognized as the velocity $(v)$ and position $(x)$. The updating rule of PSO will steer the particle swarm to gather in more promising area with better objective value.

For the $i$ th particle, let $X_{i}=\left(x_{\mathrm{i} 1}, x_{\mathrm{i} 2}, \ldots, x_{\mathrm{in}}\right)$ represents the position and $V_{i}=\left(v_{\mathrm{i} 1}, \mathrm{v}_{\mathrm{i} 2}, \ldots, v_{\mathrm{in}}\right)$ denotes the velocity, and the local best position can be written as $p_{\mathrm{i}}=\left(p_{\mathrm{i} 1}, p_{\mathrm{i} 2}, \ldots, p_{\mathrm{in}}\right)$. The global best position (guide) among all the particles can be given as $p_{\mathrm{g}}=\left(p_{\mathrm{g} 1}, p_{\mathrm{g} 2}, \ldots, p_{\mathrm{gn}}\right)$. Thus, the evolutionary strategy of the $i$ th particle in iteration $t$ can be expressed as

$$
\begin{gathered}
v_{i j}^{t+1}=w \cdot v_{i j}^{t}+c_{1} \cdot r_{1} \cdot\left(p_{i j}^{t}-x_{i j}^{t}\right)+c_{2} \cdot r_{2} \cdot\left(p_{g i}^{t}-x_{i j}^{t}\right) \\
x_{i j}^{t+1}=x_{i j}^{t}+v_{i j}^{t}
\end{gathered}
$$

where $w$ is the inertia weight factor to control particle's exploration in the searching space, $c_{1}$ and $c_{2}$ are non-negative constants called acceleration coefficients, which control the influence of $p_{\mathrm{i}}$ and $p_{\mathrm{g}}, r_{1}$ and $r_{2}$ are random numbers in the range of $[0,1]$, and $j=1,2, \ldots, \mathrm{n}$.

\section{B. Two-phase MOPSO}

For MOPSO, the goal is to find an optimal trade-off between several competing objectives for which usually no single optimal solution exists that minimizes all objective function values at the same time. As investigated in [10-11], normal means either pay attention to the convergence with worse diversity or has a better diversity but lower convergence rate, the tow-phase MOPSO method introduced in this paper takes both the convergence rate and the diversity of solutions into account. The implementation procedures are drawing as follows.

1) The Steps

Step 1: Initialize the swarm $S$ in searching space, and the PSO coefficients $w, c_{1}$ and $c_{2}$, and maximum iterations $T, t=0$.

Step 2: Initialize the position and velocity of each particle in set $S$. For the $i$ th particle, set $p_{i j}^{t}=X_{i}$ with velocity $V_{i}=0$, $i=1,2, \ldots, N$, where $N$ is the size of swarm $S$ in the archive $A$.

Step 3: For each iteration $t$, where $t=1,2, \ldots, T$,

- Select the global best position $p_{g i}^{t}$ for each particle from the archive $A$ refer to the solution's diversity.

- Update position and velocity of every particle on basis of an update equation with time variant acceleration coefficients according to the (20)-(21).

- Retain the particles within the search space in case they go beyond their boundaries. If the particle position moves beyond its boundaries, the value of its corresponding boundary will be taken.

- Update the local best position $p_{i j}^{t}$, if the current location is dominated by its local best location $p_{i}$, then the previous location is maintained, otherwise, the current location is set as the personal best location. If the particles are mutually non-dominated, one particle is selected randomly.

- Update the archive $A$ to store the non-dominated solutions from a combined population of the swarm and the archive.

- $\quad$ Loop counter points to $t+1$.

\section{2) Archive set}

Aiming to summarize a better Pareto front approaching the optimization algorithm and store particles with the best global positions, it is vital to remain the non-dominated solutions cope of the entire searching process. Archive $A$ is introduced to cumulate the non-dominated individuals from the evolutionary process in the multi-objective optimization problems.

In the starting point, the archive $A$ is set to be null and the contents will be continuous updated as the optimization proceeded by absorbing superior solutions and eliminating inferior ones. In case the archive has exceeded its marginal capacity, a crowding distance mechanism [13] is motivated to reduce the capacity of the archive $A$ without losing the diversity of the non-dominated solutions. The final crowding 
distance value of a solution is computed by adding the entire individual crowding distances in each objective function.

In particular, during the heuristic multi-objective optimization process, the algorithm is attempting to build up a discrete structure of Pareto front, which imposes the distributed solutions as diversely as possible. Since the Pareto front can be drawn in a distance metric, crowding distance will be calculated for each candidate solution separately. Here, a solution with a longer crowding distance means it is more crowded than other solutions and the diversity of the solution is higher. Consequently, the top ranking of such sort of solutions should be considered as the optimal solution.

\section{3) Two-phase MOPSO strategy}

As proposed in [14], the two-phase MOPSO consists with

- The $1^{\text {st }}$ phase, where the Sigma method [10] is employed to facilitate the fast convergence and obtain an approximated Pareto front.

- $\quad$ The $2^{\text {nd }}$ phase, where an ideal optimal particle method is adopted to promote the diversity of the solution. For a multi-objective optimization problem, the ideal optimal particle $X^{*}$ is decided by

$$
\min \varphi\left(f\left(X^{*}\right)\right)=\sqrt{\sum_{k=1}^{n}\left(f_{k}\left(X^{*}\right)-f_{k}^{*}\right)^{2}}
$$

where $f_{k}^{*}$ is the previous optimal value of the $k$ th objective, $k=1,2, \ldots K$.

The selection of an appropriate guide particle is the most crucial work in MOPSO process, a proper guide is the key to achieve better convergence among non-dominated solutions towards the Pareto front associated with better diversity characteristics. Several guide selection mechanisms have been conducted by [9], [15], such as the dynamic neighborhood, the non-dominated sorting PSO, the roulette wheel selection and random selection, the sub-swarm-based method, Sigma method, SPEA2-based guide method and HSG-MOPSO method. In this paper, the procedures of selecting the guide particles are concluded as,

- A set of potential guides is created and updated in each iteration $t$ using the available non-dominated solutions in archive $A$.

- Calculate the optimal value $f_{k}^{*}$ and the corresponding particle $X_{i}^{f}$, respectively.

- $\quad$ Gain the ideal optimal particle $X^{*}$ by (22).

- Calculate the guide $p_{g}^{*}$ according to the ideal optimal particle $X^{*}$, where $p_{g}^{*}$ is the optimal value for each objective $k$.

To reduce the MOPSO computational burden and make it easier to be implemented, a mutation operator introduced in [9] is involved to ensure the algorithm converging in the global optimal front.

\section{MOTP program flow}

Considering the MOPSO approach is essentially an unconstrained optimization algorithm, a brief scheme [11] is added to deal with the constraints of the proposed MOTP problem, i.e. if two individuals are compared and feasible due to their constraints, non-dominance is directly utilized to choose which is better. If two of them are both infeasible, the one with the lowest amount of constraint violation host the other. If one is feasible and the other is infeasible, the feasible one dominates.

The major MOTP modules and the general flow of the program are shown in Figure 1. The Fuzzy satisfying decision making approach introduced in this program has been discussed in [7], which consists of three major steps, i.e. boundary decision, normalization and decision analysis.

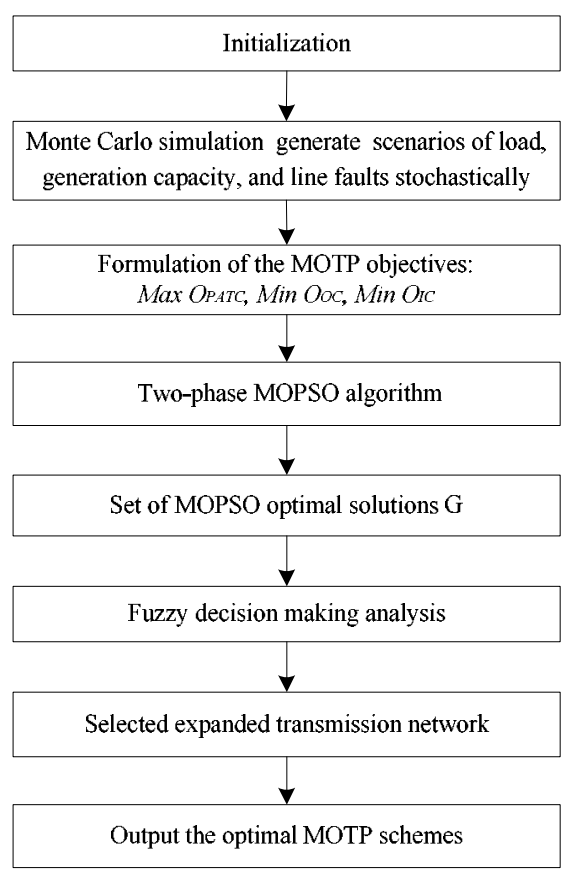

Figure 1. MOTP program flow

\section{CASE Study}

Case study has been carried out on the 77-bus system, to prove the effectiveness of both the proposed multi-objective planning approach and two-phase MOPSO. The parameter details of the 77-bus system can be found in [3]. The system has 93 right-of-ways, the active power transmission limit of each line is $2300 \mathrm{MW}$, and line cost is $\$ 193,200 / \mathrm{km}$.

The best three planning schemes of the 77-bus system are presented in detail in Figure 2 and TABLE I, which all indicate the network of the 77-bus system has a relative tightly linked structure. Further comparison between $S_{1}$ and $S_{2}$ shows that, under the almost same power outage cost, $S_{1}$ is definitely better than $S_{2}$ due to its higher PATC and lower investment cost. Compared with $\mathrm{S}_{3}, \mathrm{~S}_{1}$ has extremely high PATC and obviously low cost of power outage but with a notable increase on the investment cost. 


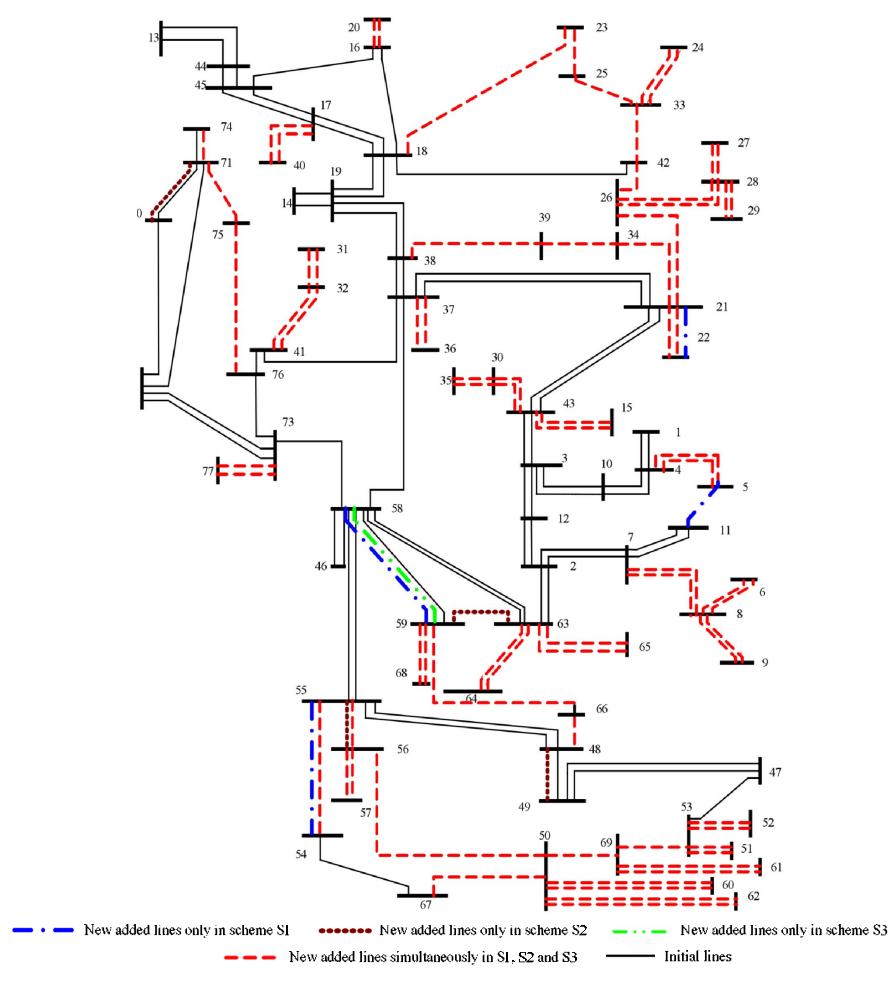

Figure 2. Topology of the expanded network of the 77-bus system

TABLE I. Best Planning Schemes of The 77-Bus System

\begin{tabular}{|c|c|c|c|}
\hline Schemes & $\mathbf{S}_{1}$ & $\mathbf{S}_{2}$ & $\mathbf{S}_{3}$ \\
\hline $\begin{array}{c}\text { Same new } \\
\text { added lines }\end{array}$ & \multicolumn{3}{|c|}{$\begin{array}{l}6-8(2), 7-8(2), 8-9(2), 15-43(2), 16-20(2), 17-40(2), \\
18-23(1), 21-22(2), 21-26(1), 21-34(1), 23-25(1), 24- \\
33(2), 25-33(1), 26-28(2), 27-28(2), 28-29(2), 30- \\
35(2), 30-43(2), 31-32(2), 32-41(2), 33-42(1), 34- \\
39(1), 36-37(2), 38-39(1), 50-60(2), 50-62(2), 50- \\
67(1), 50-69(1), 51-53(2), 52-53(2), 53-69(1), 55- \\
56(1), 56-57(2), 59-68(2), 61-69(2), 63-64(2), 63- \\
65(2), 73-77(2), 4-5(2), 26-42(1), 48-66(1), 50-56(1), \\
54-55(1), 59-66(1), 71-74(1), 71-75(1), 75-76(1)\end{array}$} \\
\hline $\begin{array}{l}\text { Different new } \\
\text { added lines }\end{array}$ & $\begin{array}{c}5-11(1), 21- \\
22(1), 54-55(1) \\
58-59(1)\end{array}$ & $\begin{array}{c}48-49(1), 55- \\
56(1), 59-63(2), \\
70-71(1)\end{array}$ & $58-59(1)$ \\
\hline $\operatorname{PATC}(\%)$ & 18.4 & 17.9 & 9.7 \\
\hline $\begin{array}{c}\text { Cost of power } \\
\text { outage }\left(\times 10^{4} \$\right)\end{array}$ & 71.5 & 69.8 & 103.3 \\
\hline $\begin{array}{c}\text { Investment cost } \\
\left(\times 10^{4} \$\right)\end{array}$ & 105210.0 & 105712.6 & 72501.3 \\
\hline
\end{tabular}

V. CONCLUSIONS

A MOTP approach considering uncertainties is proposed in this paper, the objectives are PATC, the cost of power outage and the network investment cost. The solving algorithm based on the two-phase MOPSO is also introduced. The planning results of the 77-bus system show that, for a large and practical system, the proposed MOTP method can effectively enhance the transmission ATC by adding specific new lines under the variety conditions of uncertainties. Considering efficiency, reliability, and economic, the best planning schemes can be put forward by the two-phase MOPSO, which shows its superiority as well.

Further research can focus on the multi-stage and multiobjective model, which should consider the uncertainty of the bidding parameters and other uncertain factors in transmission expansion problem.

\section{ACKNOWLEDGMENT}

The authors gratefully acknowledge the financial supports and the strategic platform for innovation \& research provided by Danish national project iPower.

\section{REFERENCES}

[1] J. H. Zhao, Z. Y. Dong, P. Lindsay, and K. P. Wong, "Flexible Transmission Expansion Planning With Uncertainties in an Electricity Market," IEEE Trans. Power Systems, vol. 24, pp. 479-488, Feb. 2009.

[2] D. Delgado, and J. Claro, "Transmission network expansion planning under demand uncertainty and risk aversion," International Journal of Electrical Power \& Energy Systems, vol. 44, pp. 696-702, Sep. 2012.

[3] Y. Wang, H. Z. Cheng, C. Wang, and et al, "Pareto optimality-based multi-objective transmission planning considering transmission congestion," International Journal of Electrical Power \& Energy Systems, vol. 78, pp. 1619-1626, Mar. 2008.

[4] E. B. Cedeño, and S. Arora, "Performance comparison of Transmission Network Expansion Planning under deterministic and uncertain conditions," International Journal of Electrical Power \& Energy Systems, vol. 33, pp. 1288-1295, Jun. 2011.

[5] T. Akbari, A. Rahimi-Kian, and M. T. Bina, "Security-constrained transmission expansion planning: A stochastic multi-objective approach," International Journal of Electrical Power \& Energy Systems, vol. 43, pp. 444-453, Jul. 2012.

[6] M. O. Buygi, H. M. Shanechi, G. Balzer, and M. Shahidehpour, "Market based transmission expansion planning," IEEE Trans. Power Systems, vol. 19, pp. 2060-2067, Feb. 2004.

[7] T. S. Chung, K. K. Lee, G. J. Chen, J. D. Xie, and G. Q. Tang, " Multiobjective transmission network planning by a hybrid GA approach with fuzzy decision analysis," International Journal of Electrical Power \& Energy Systems, vol. 25, pp. 187-192, Jun. 2003.

[8] R. Poli, J. Kennedy, and T. Blackwell, Particle swarm optimization an overview, vol. I. MIT: Wiley, 2010, p. 2.

[9] A M. R. Sierra, and C. A. Coello, "Multi-objective particle swarm optimizers: A survey of the state-of-the-art," International Journal of Computational Intelligence Research, vol. 3, pp. 287-308, Jul. 2006.

[10] S. Mostaghim, J. Teich, "Strategies for finding good local guides in multi-objective particle swarm optimization (MOPSO)," in Proc. 2003 IEEE Swarm Intelligence Symposium, pp. 26-33.

[11] M. S. Lechuga, and C. A. Coello, "Handling multiple objectives with particle swarm optimization," IEEE Trans. Evolutionary Computation, vol. 3, pp. 256-279, Mar. 2006.

[12] L. Goel, R. Gupta, and M. F. Ercan, "Including higher order outages and well-being considerations in sub-transmission system reliability worth assessment," IEEE Trans. Power Systems, vol. 60, pp. 39-47, Sep. 2001

[13] K. Deb, A. Pratap, S. Agarwal, and T.Meyarivan, "A fast and elitist multiobjective genetic algorithm: NSGA-II," IEEE Trans. Evolutionary Computation, vol. 6, pp. 182-197, Apr. 2002.

[14] G. Hu, Z. Mao, and D. He, "Multi-objective optimization for leaching process using improved two-stage guide PSO algorithm," J. Cent. South Univ. Technol., vol. 18, pp. 1200-1210, 2011.

[15] S. Ganguly, N. C. Sahoo, and D. Das, " Multi-objective particle swarm optimization based on fuzzy-Pareto-dominance for possibilistic planning of electrical distribution systems incorporating distributed generation," Fuzzy, vol. 213, pp. 47-73, 2013. 